\title{
The observations of Near Earth Objects by the automatic mirror astrograph $\mathrm{ZA}-320 \mathrm{M}$ at Pulkovo observatory
}

\author{
A. V. Devyatkin, A. P. Kulish, V. V. Kouprianov, D. L. Gorshanov, \\ A. S. Bekhteva, O. V. Krakosevich, E. Yu. Aleshkina, F. M. \\ Ibragimov, V. N. L'vov, R. I. Smekhacheva and S. D. Tsekmeister \\ Main (Pulkovo) Astronomical Observatory of Russian Academy of Science
}

\begin{abstract}
At Pulkovo Observatory, we conduct observations of various Solar System bodies: major planets, their satellites, comets, and asteroids, including Near Earth Objects. For these purposes, a robotic telescope was constructed on the base of the ZA-320 Mirror Astrograph $(D=320 \mathrm{~mm}, F=3200 \mathrm{~mm})$. It can perform CCD observations of Solar System bodies with the limiting magnitude of up to 19.0 .

Independent ephemeris support is provided by the EPOS software package developed at Pulkovo Observatory; it includes tracing of catalogues of comets and asteroids, regular ephemeris calculations, and control of observations. CCD frame processing is done by the Apex automatic data reduction package developed at Pulkovo Observatory.

In 2001-2006, more than 12000 observations of minor Solar System bodies were collected, including more than 6000 positions of 656 NEAs, about 1200 observations of 27 comets, and about 2000 observations of major planets satellites. The mean accuracy of obtained positions is $0^{\prime \prime} .09-0^{\prime \prime} .40$. Results of observations are regularly submitted to the Minor Planet Center.

Currently, ZA-320M is the 16-th of more than 680 telescopes in the worldwide rating of those that observe NEAs (by the number of observations).

In the near future, our group is planning to start observations with another two robotic telescopes: MTM-500 ( $D=500 \mathrm{~mm}, F=4000 \mathrm{~mm}$ Maksutov) and 1-meter telescope $(D=$ $1000 \mathrm{~mm}, F=1200 \mathrm{~mm}$ ) of the Pulkovo mountain station at Northern Caucasus (Kislovodsk, $2100 \mathrm{~m}$ above sea level). These two instruments will allow to increase the number of observations, their accuracy, and limiting magnitude (up to $20.5 \mathrm{mag}$ ).
\end{abstract}

Keywords. Astrometry; follow up; photometry; telescope

\section{Introduction}

The ZA-320 Mirror Astrograph ( $D=320 \mathrm{~mm}, F=3200 \mathrm{~mm}$ ) has been working at Pulkovo Observatory (Saint-Petersburg, Russia) since 1997. The telescope is used for various kinds of astrometric and astrophysical CCD observations. The following list presents celestial objects observed by our telescope.

I. Asteroids:

(a) Near Earth Asteroids

(b) Recently discovered asteroids

(c) Other asteroids from the MPC Critical List

(d) Double asteroids

(e) Asteroids - extinct comets (3200 Phaethon)

(f) Close approaches of asteroids

(g) Visual approaches of asteroids

(h) Asteroids - space mission targets (25143 Itokawa) 
(i) Stellar occultations by asteroids (111 Ate, 2000)

II. Comets

III. Satellites of giant planets:

(a) Jupiter: Himalia, Elara, Pasiphae

(b) Saturn: Titan, Hyperion, Iapetus, Phoebe

IV. Planets (and dwarf planets):

(a) Jupiter: Himalia, Elara, Pasiphae

(b) Saturn: Titan, Hyperion, Iapetus, Phoebe

V. Objects of the geosynchronous Earth orbit

VI. Lagrange points of Earth

VII. Photometric observations:

(a) Asteroids, comets, satellites of giant planets

(b) Star with "eclipsing" planet - HD209458

(c) Mutual phenomena in systems of satellites of Jupiter and Saturn

(d) $\mathrm{SNe}$

(e) Variable stars

(f) Open clusters

Still the primary goal of the telescope includes astrometric observations of asteroids and comets, including Near Earth Objects.

Up to 2005 we used a small-size CCD camera SBIG ST-6 $\left(375 \times 242\right.$ pix, $9^{\prime} .5 \times 7^{\prime} .5$ field of view). Since 2005 , we use FLI IMG1001E CCD camera $\left(1024 \times 1024\right.$ pix, $\left.28^{\prime} \times 28^{\prime}\right)$.

The ZA-320M telescope control system (TCS) includes capabilities for remotely controlling the telescope, as well as for the fully automatic operation without any human intervention. TCS is able to control pointing, dome and slit, filter wheel, CCD camera, focuser, autoguider, and to perform observation scheduling via the built-in ephemeris interface. Another software package is used for automatic processing of raw observation results.

The TCS software consists of the following main modules:

- telescope control subsystem,

- image acquisition and CCD camera control subsystem,

- ephemeris support,

- reduction of CCD-frames.

\section{Telescope control subsystem}

The TCS software for the ZA-320M robotic telescope has the distributed modular design and thus can be used to control various types of telescopes. In the following we briefly describe the major TSC modules.

High precision clock interface. Provides accurate high-resolution UTC time for all TCS modules. Various time sources, like GPS receivers and synchronization signal from atomic standards, are supported. Implemented as a standalone package (AccuTime) including the software development kit $(\mathrm{SDK})$ for $\mathrm{C} / \mathrm{C}++$, Ada95, Delphi, and Python programming languages, with easy integration into other software packages and customization to various time sources.

Distributed datalogging service. Provides a centralized datalogging facility based on the client-server approach, with a simple application programming interface (API) allowing all TCS components running on any of the local control units (LCUs) of the ZA-320M network to register events in the global journal. 
TCS hardware layer. Implements a number of high-level commands, like pointing to the specified target, tracking control, dome control, etc., for the actual TCS hardware protocols.

Operator interface. Provides the graphical user interface (GUI) for planning observations, watching the current TCS state, and manual control of the telescope operation. Automatic object scheduler can choose the sequence of observed objects to achieve the optimal observation conditions for every object, taking into account their priorities and possible constraints and requirements. TCS can operate in the manual, semi-automatic, and fully automatic modes.

Image acquisition subsystem. ZA-320M image acquisition subsystem is a stand-alone software package CameraControl designed for controlling various types of CCD cameras via the graphical user interface (GUI). It is fully integrated into the whole TCS package and is able to operate as a pure image acquisition module. Like all other TCS components, CameraControl is based on the modular design. Dedicated modules implement a generic set of high-level commands, like cooler, shutter, and exposure control, and image downloading, for the particular CCD camera.

Apart from the built-in FITS format support using the CFITSIO library, various image storage formats are supported via extension module API. Basic image examination facilities, including brightness and contrast control, histogram equalization, magnifying glass, 2D and 3D profile visualization, area statistics, equatorial grid etc., are included into the GUI.

CameraControl TCS integration is based on the built-in Python interpreter with a full set of commands for operating the camera and querying its state, and on the integrated TCP server for remote camera control. Other TCS modules, like observation scheduler, working as clients, can thus obtain the full control over the image acquisition process, though the capability of performing exposures manually is still left intact.

\section{Ephemeris support - the EPOS software}

EPOS (Ephemeris Program for Objects of the Solar system) is the effective application for study and ephemeris support of observations of the Solar systems objects. This program was developed at Pulkovo Observatory. The orbital elements of asteroids and comets as well as the observatories' coordinates obtained via Internet are used by EPOS. The applications operation is also based on the data of modern numerical ephemerides (DE200/LE200, DE405/LE405, DE406/LE406) and star catalogs (Hipparcos, Tycho-2, USNO, etc.) that are distributed on the CDs. EPOS is intended for use under Windows 98/XP and has bilingual (English and Russian) interface. EPOS (version 5.x) includes the following components:

The main program. It transfers the control to one of the programs that solve the restricted set of tasks. This program also imports the observatories' data and controls the use of various numerical ephemerides.

"Catalogs of objects". This program stores in the internal database the orbital elements and other parameters of the minor bodies of the Solar system (now more than 300000 asteroids and more than 500 comets). This helps to browse, edit and export these data, to select the objects with respect to various conditions and to import the most recent data from the well known catalogs (B.Marsden, E.Bowell). One can get the histograms and distributions for various parameters. It is possible to work with numerous catalogs of objects (for example, NEAs, trans-Neptunians, Centaurs, Trojans and Hildas, etc.).

"Ephemerides". This program calculates the Solar system objects' ephemerides of various type and accuracy. The calculated values can be observable and geometric 
coordinates, and the osculating orbital elements. The observable are astrometric or apparent coordinates along with their first two derivatives or Pulkovo parameters of apparent motion and a set of other values. The advantage of this program is the ability to create the list of necessary parameters located in the required order and properly formatted. The geometric are the rectangular and spherical coordinates of any object referred to the centers of the Sun, Moon or major planets. The ephemerides may be calculated with various accuracy, from elliptic motion to the high precision numerical integration of equations of motion taking into account the perturbations from all major planets and some minor bodies. One can use the values of orbital elements or rectangular coordinates that are placed to the special input files with higher formal accuracy. Various combinations of elements and some group operations are possible in the ephemeris calculation.

"O $-C$ : Comparison of Observations with Calculations". This program compares observed coordinates and velocities with the calculated ones. This helps to evaluate the observational accuracy, to reveal the large errors, to identify the objects. Thus an observer can control the results of observations before sending them to Minor Planet Center.

"Frame". The program visualizes the apparent motion of many objects on the star background and their diurnal motion as well. One can get the list of objects that are observable at the specified moment in the specified sky area or even in the space box. This helps in identification of objects and in the study of the structure of the Solar system.

"Orbits". This program visualizes the perturbed motion of many objects and groups of objects along their heliocentric orbits in the space. It is possible to vary the observers position, the scale, the velocity and direction of motion, to switch on/off the numbers and names of the objects, their orbits, apsidal and nodal lines and the ecliptic plane. The picture may be saved in BMP or EMF files. The useful quality of the program is the opportunity to accumulate the images of the lines that helps to follow the orbital evolution in time.

"Hazardous Objects". By this program one can get the current list of PHA - potentially hazardous objects for the Earth and other major planets, and the list of close approaches of asteroids to the specified major planet within the specified time span.

"What to Observe". The program generates the list of objects observable at the specified place in the specified night. One can limit the values of visual magnitude, the objects altitude and elongation from the Sun.

So with the EPOS software package one can calculate the accurate ephemerides for many objects and get the quick illustration of their motion as well. This helps to support the existing observational programs, to develop the new ones and to put the ephemeris data at the disposal of the interested observers and publishers.

EPOS was used in research of the dynamical structures of the Solar system, in preparation and analysis of Pulkovo observations of asteroids and comets, 6-meter BTA observations of trans-Neptunian objects, in support of NEA international radar observations, in the accuracy analysis of asteroidal observations of many observatories throughout the world and in other tasks.

\section{Processing of CCD frames - Apex software package for data reduction}

Observational data are being processed using the Apex image processing software series - the former semi-automatic package Apex 1.0 and the recent fully automatic pipeline based on the Apex II platform, both developed at Pulkovo observatory.

Apex $I I$ is a general-purpose software platform for astronomical image processing. Its architecture and design concepts are similar to those of the major image processing 
packages including IRAF, MIDAS, and IDL. Like them, Apex II consists of several components:

- core - high-level interpreted dynamic (scripting) programming language;

- standard library of general-purpose utility functions and algorithms specific to the area of astronomical image processing;

- object-oriented graphical user interface (GUI) subsystem with interactive image examination and data visualization capabilities, built on top of the core language and library;

- a set of user functions and scripts which utilize the above components to perform particular image processing tasks.

This structure has proven to be most flexible and versatile. It allows implementing the full range of image processing applications - from interactive command-line driven tools with interactive examination of intermediate processing results to fully automated pipelines for processing large data volumes to stand-alone GUI applications for specific image reduction tasks.

Unlike the image processing packages mentioned above, Apex II is not based upon a dedicated interpreted programming language, but rather upon the widely-used generalpurpose object-oriented scripting language Python. This choice is motivated primarily by the clarity, power, and flexibility of the language, existence of implementations for all major hardware and software platforms, and the extensive standard library for most routine tasks like input/output, data visualization, matrix algebra, curve and surface fitting, n-dimensional image processing etc. Despite the widespread opinion about the low performance of scripting languages, pure Python scripts in Apex II are often faster than similar programs written in conventional compiled programming languages. This is mostly due to the high level of vectorization of mathematical operations and to effective optimization of underlying $\mathrm{C} /$ Fortran libraries.

All these advantages currently attract attention of the leading scientific software developers. The evidence for this are Python interfaces to the two major astronomical image processing systems, PyRAF and, recently, PyMIDAS.

The standard Apex II library is built primarily on top of the two Python packages, Numerical Python and Scientific Python (NumPy/SciPy). The first of them implements the basic functionality for working with multidimensional arrays, including vectorization and matrix algebra. The second one provides implementation of most of the algorithms commonly used in scientific applications: Fourier transform, integration, solving PDEs, interpolation, optimization and nonlinear regression, signal and image processing, special functions etc. Based on these algorithms, as well as on the built-in Python functions, the Apex II library implements various higher level tasks specific to the field of astronomical image processing, like timescale conversions, calibration and filtering of CCD images, automatic object detection, PSF fitting, astrometric and photometric reduction, catalog access and so forth.

The graphical subsystem (still under active development) is based on wxWidgets/ wxPython, the cross-platform GUI toolkit, and on matplotlib, the scientific data visualization package modeled after MATLAB. These packages can be used to display individual CCD frames or catalog fields, plot various data obtained during image processing, as well as create standalone GUI applications intended for processing of specific kinds of astronomical images.

Thus Apex II is primarily a general-purpose software platform for development of reduction systems for various astronomical data.

Currently, the Apex II platform is used primarily for automated astrometric and photometric reduction of large volumes of observational data for various Solar system objects. 
The dedicated image processing pipeline is capable of producing reports in the standard Minor Planet Center format, as well as estimating the accuracy of observations with the help of the EPOS software package. Astrometric reduction is performed in the system of the USNO-A2.0, USNO-B1.0, UCAC-2 catalogs, accounting for chromatic refraction.

The main Apex II image processing pipeline for Solar system objects includes the following steps:

- automatic selection (and, if necessary, creation) of required calibration frames for each frame being processed; bias/dark/flat correction;

- estimation and subtraction of sky background;

- optimal image filtering for increasing the objects signal-to-noise ratio (SNR);

- object extraction by thresholding with logical filtering intended to increase the probability of detection of faint objects and to reduce the number of false detections;

- deblending of overlapping objects;

- finding the objects centroids by PSF fitting;

- flux measurement using the aperture, PSF, or optimal photometry techniques;

- elimination of the remaining false detections (including cosmic rays) using the various criteria;

- reference astrometric catalog matching, with the possibility to find the target field in the catalog area of the given size;

- astrometric reduction - obtaining the LSPC solution using the selected plate model;

- reference photometric catalog matching and differential photometry in the instrumental band;

- post-identification of all unknown objects within the frame using all available ephemeris databases and other catalogs;

- generation of the standard MPC report for all Solar system objects within the frame.

This pipeline allows to perform reduction of large volumes of raw observational data without sacrificing accuracy. The possible manual operations here reduce to the final examination of series of measurements to eliminate the possible processing errors that are hard to detect by any formal criteria.

\section{Results: Astrometry}

The "Pulkovo Program for Research of Near Earth Objects" started in 2002 using the ZA-320M telescope. During 2001-2005, more than 12000 observations of minor Solar System bodies were performed, including more than 6000 observation of 656 NEAs and about 1200 observations of 27 comets. These observations were processed in the framework of the USNO-A2.0, USNO-B1.0, and UCAC-2 catalogues. The mean accuracy of the obtained positions is $0 .{ }^{\prime \prime} 09-0 . .^{\prime \prime} 40$.

The results of the observations are regularly submitted to the Minor Planet Center. According to the Center information, the ZA-320M telescope is rated 16 -th by the number of observations in the world-wide list of more than 680 telescopes that observe NEAs.

In the following table, we highlight several examples of our astrometric results. Here $N$ is the number of observations for the object, $\overline{(O-C)_{\alpha} \cos \delta}$ and $\overline{(O-C)_{\delta}}$ are mean values of $(O-C)$ difference for the whole observational period, for right ascension and declination, respectively; $\sigma_{\alpha} \cos \delta$ and $\sigma \delta$ are accuracies of a single observation for right ascension and declination, respectively (estimation by the $(O-C)$ ); $\Delta m$ is the magnitude range of the object during observational period. 


\begin{tabular}{l|c|c|c|c|c|c}
\hline \multicolumn{1}{c}{ Object } & $N\left|\overline{(O-C)_{\alpha} \cos \delta}\right| \sigma_{\alpha} \cos \delta$ & $\overline{(O-C)_{\delta}}$ & $\sigma \delta$ & $\Delta m$ \\
\hline 9 Metis & 46 & $+0 .{ }^{\prime \prime} 38$ & $\pm 0 .{ }^{\prime \prime} 18$ & $+0 .^{\prime \prime} 42$ & $\pm 0 .{ }^{\prime \prime} 24$ & $8.6-10.9$ \\
433 Eros & 65 & +0.14 & 0.27 & +0.43 & 0.28 & $11.4-12.6$ \\
1866 Sisyphus & 24 & 0.00 & 0.24 & +0.19 & 0.29 & $15.1-17.7$ \\
3122 Florence & 18 & -0.12 & 0.23 & +0.19 & 0.25 & $15.9-17.7$ \\
3199 Nefertiti & 27 & +0.14 & 0.40 & +0.18 & 0.35 & $15.5-17.9$ \\
3200 Phaethon & 18 & 0.00 & 0.40 & +0.03 & 0.37 & $16.3-18.4$ \\
4179 Toutatis & 10 & -0.17 & 0.42 & +0.39 & 0.24 & $14.8-16.8$ \\
2004 FX31 & 22 & +0.05 & 0.46 & -0.02 & 0.50 & $17.3-18.9$ \\
2004 JA & 33 & -0.01 & 0.21 & -0.09 & 0.17 & $15.6-16.8$ \\
2P Encke & 7 & +0.33 & 0.51 & +0.72 & 0.24 & $14.5-16.3$ \\
1036 Ganymed & 44 & -0.21 & 0.20 & +0.02 & 0.12 & $13.3-13.9$ \\
3800 Karayusuf & 41 & -0.35 & 0.12 & -0.31 & 0.08 & $16.0-17.0$ \\
423 Diotima & 70 & -0.10 & 0.16 & -0.06 & 0.10 & $11.1-12.9$ \\
5164 Mullo & 34 & -0.08 & 0.10 & -0.03 & 0.07 & $16.4-17.4$ \\
85709 & 45 & -0.28 & 0.12 & -0.27 & 0.07 & $15.5-16.6$ \\
C/2003 WT42 & 24 & +0.89 & 0.09 & -0.32 & 0.06 & $15.5-16.3$ \\
\hline
\end{tabular}

\section{Results: Photometry}

Photometric measurements of minor bodies of the Solar System are made along with their astrometric measurements. Photometric observations are made in the Johnson BVR bands, as well as in the wide instrumental band of the telescope (350-1000 nm) - in the cases of weak objects and when very short exposure time is needed. For photometric processing of asteroids and comets, we use USNO-A2.0, USNO-B1.0, and UCAC-2 catalogues.

We obtain photometry only for asteroids that demonstrate fast magnitude changes. For example, NEA 1999 HF1 has shown variability with characteristic time less then half an hour. A number of series of its observations were made on several nights. They have shown that its magnitude changes with the quasi-period of about 20 minutes. The amplitude of magnitude changes from night to night lies in the range of $0 .^{m} 1-0 .^{m} 4$. Mean accuracy of photometry in these observations was about $0 .{ }^{m} 06$. The following figure shows an example of the $1999 \mathrm{HF} 1$ observation series.

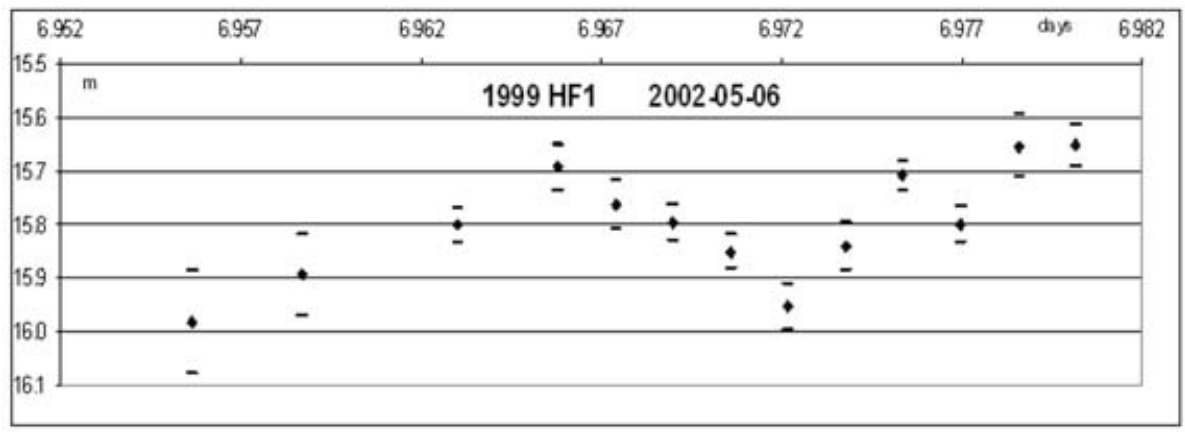




\section{Conclusions}

The experience gained in the course of construction of the ZA-320M robotic telescope is used now for modernization of the two other telescopes: Maksutovs MTM-500 ( $D=$ $500 \mathrm{~mm}, F=4000 \mathrm{~mm})$ and 1-meter telescope $(D=1000 \mathrm{~mm}, F=1200 \mathrm{~mm})$. We are planning to mount MTM-500 on the mountain observational site of Pulkovo Observatory at Northern Caucasus (Kislovodsk, $2100 \mathrm{~m}$ above sea level) in 2007. The telescope will be used for astrometric and photometric observations of asteroids, comets and other Solar System bodies. We are planning that these instruments will produce a significant increase in the number of observations, their accuracy, and limiting magnitude (up to 20.5 mag).

\section{References}

Devyatkin, A. V. \& Gorshanov, D. L. 2001, IBVS 5072

Devyatkin, A. V., Gorshanov, D. L., Gritsuk, A. N., Mel'nikov, A. V., Sidorov, M. Yu., \& Shevchenko, I. I. 2002, Solar System Research 3, 248

Devyatkin, A. V., L'vov, V. N., Kornilov, E. V., Gorshanov, D. L., Kouprianov, V. V.,Sidorov, \& M. Yu. 2002, Izvestia GAO 216, 120 (in Russian)

Devyatkin, A. V., L'vov, V. N., Gorshanov, D. L., Kouprianov, V. V., Aleshkina, E. Yu., Bekhteva, A. S., Baturina, G. D., Kornilov, E. V., \& Sidorov, M. Yu. 2004, Izvestia GAO 217, 236 (in Russian)

Devyatkin, A. V., Aleshkina, E. Yu., Barshevich, K. V., Baturina, G. D., Bekhteva, A. S., Gorshanov, D. L., Krakosevich, O. V., Kouprianov, V. V., L'vov, V. N., Smekhacheva, R. I., Sochilina, A. S., \& Tsekmejster, S. D. Proc. of Sixth US/Russian Space Surveilence Workshop (August 22-25, 2005, Pulkovo, St.-Petersburg, Russia), 2005, p. 220

Frolov, V. N., Jilinski, E. G., Ananjevskaja, Y. K., Bronnikova, N. M., Poljakov, E. V., \& Gorshanov, D. L. 2002, A\&\&A 369, 115

Frolov, V. N., Ananjevskaja, J. K., Jilinski, E. G., Gorshanov, D. L., \& Bronnikova, N. M. 2006, $A \mathscr{E A} 451,901$

Kanaev, I. I., Devyatkin, A. V., Kulish, A. P., Rafalskiy, V. B., Vinogradov, V. S., Kouprianov, V. V., \& Kornilov, E. V. 2004, Izvestia GAO 217, 505 (in Russian)

Kanaev, I. I., Sochilina, A. S., L'vov, V. N., Devyatkin, A. V., Kouprianov, V. V., Gorshanov, D. L., Sidorov, M. Yu., Guseva, I. S., \& Molotov, I. E. 2003, Proc. of Fifth US-Russian Space Surveillance Workshop, (24-27 September, 2003, Pulkovo, St.-Petersburg, Russia) p. 6

Minor Planet Electronic Circ. No. 2003-M40, 2003-H21, 47449, 47506, 47994, 48317, 48619, 49222, 49389, 49426, 50321 